\title{
A comparative study of Mycobacterium avium subsp. avium and Mycobacterium avium subsp. hominissuis in experimentally infected pigs
}

\author{
Angelika Agdestein ${ }^{1 *}$, Tone B Johansen ${ }^{1}, \varnothing y v o r$ Kolbjørnsen ${ }^{1}$, Anne Jørgensen², Berit Djønne ${ }^{1}$ and Ingrid Olsen ${ }^{1}$
}

\begin{abstract}
Background: Mycobacterium avium subsp. avium (Maa) and Mycobacterium avium subsp. hominissuis (Mah) are opportunistic pathogens that may infect several species, including humans and pigs. Mah is however more frequently isolated from pigs than Maa, and it is unclear if this is due to difference in virulence or in exposure to the two organisms. Clinical isolates of each subspecies were administered perorally to ten domestic pigs, respectively. The animals were sacrificed at six and 12 weeks after inoculation. At necropsy, macroscopic lesions were recorded, and tissue samples were collected for mycobacterial culture, IS1245 real time PCR and histopathological examination. Culturing was also performed on faecal samples collected at necropsy.

Results: Macroscopic and histopathological lesions were detected in pigs infected with each subspecies, and bacterial growth and histopathological changes were demonstrated, also in samples from organs without gross pathological lesions. Six weeks after inoculation, live Mah was detected in faeces, as opposed to Maa. The presence of live mycobacteria was also more pronounced in Mah infected tonsils. In comparison, the Maa isolate appeared to have a higher ability of intracellular replication in porcine macrophages compared to the Mah isolate.

Conclusions: The study shows that both subspecies were able to infect pigs. Additionally, the more extensive shedding of Mah might cause pig-to-pig transmission and contribute to the higher incidence of infection caused by this subspecies.
\end{abstract}

Keywords: Mycobacterium avium, Experimentally infected pigs, Transmission, Source of infection

\section{Background}

The genus Mycobacterium covers a broad spectrum of acid-fast staining species, ranging from harmless saprophytes to significant pathogens [1]. M. avium subsp. avium (Maa) and M. avium subsp. hominissuis (Mah) are two subspecies of M. avium, subordinated the Mycobacterium avium complex (MAC) [2]. Mah can cause serious systemic infection in immunocompromised patients, such as humans infected with HIV [3]. Additionally, this opportunistic pathogen can cause cervical lymphadenitis in children and lung infections in patients with underlying lung disease [1]. In pigs, Mah is frequently responsible for lesions in lymph nodes of the digestive tract, and can also lead to systemic infection

\footnotetext{
* Correspondence: angelika.agdestein@vetinst.no

${ }^{1}$ Norwegian Veterinary Institute, PO. Box 750 Sentrum, N-0106 Oslo, Norway Full list of author information is available at the end of the article
}

with affection of parenchymatous organs. Since Mah usually does not cause clinical signs in pigs, the lesions are mainly detected at slaughter [4]. Maa is the causative agent of tuberculosis in birds, in which it acts as an obligate pathogen, causing contagious, chronic disease $[5,6]$. Maa has also been isolated from humans and pigs with mycobacteriosis $[7,8]$.

Before molecular typing enabled differentiation of Maa and Mah isolates, birds were believed to be an important source of mycobacteriosis in pigs [9]. However, various molecular investigations on clinical isolates have shown this is rather the exception than the rule $[7,10,11]$. Although $M a a$ has been reported in pigs and in humans $[7,8]$, Mah plays the key role in MAC infections in these mammalian species [12-16]. In Norway only Mah has been detected in pigs [17]. Peat and sawdust have proved to be important sources of porcine Mah infection by
Ciomed Central

() 2012 Agdestein et al; BioMed Central Ltd. This is an Open Access article distributed under the terms of the Creative Commons Attribution License (http://creativecommons.org/licenses/by/2.0), which permits unrestricted use, distribution, and reproduction in any medium, provided the original work is properly cited. 
various authors $[11,18-20]$, whereas human infection often is contracted from water $[6,21]$. The cause of the discrepancies of the prevalence of each subspecies with regards to hosts remains unclear. Suggested factors are sampling procedures, differences in farming conditions and environmental exposure [8]. Differences between $M a a$ and Mah with regards to virulence and susceptibility in pigs might also be important factors contributing to the dominant presence of Mah. Infections with $M$. avium might be of zoonotic importance [4,22], and the possibility of pigs being a source for human infection cannot be ruled out $[10,16]$. More knowledge about routes of transmission in animals and humans is required for control of the disease $[3,13]$.

Experimental infection of pigs with $M$. avium prior to the division of subspecies avium into Maa and Mah has been described in the literature [9,23-27]. After the application of the new systemic nomenclature, Maa infection has been characterized in wild boars by experimental infection [28]. Additionally, collection strains of $M a a$ and Mah were compared in a study aiming to optimise diagnostic methods [29].

The aim of the present study was to elucidate whether $M a a$ and Mah have a different potential to infect pigs that may explain the differences in prevalence between the two subspecies. The results showed a slightly, but not significantly, elevated ability of Mah to infect pigs, compared to Maa. The main finding, however, was the event of faecal shedding of Mah at 6 weeks after infection, which was not observed in pigs infected with Maa.

\section{Methods \\ Animals}

The use of experimental animals was approved by the Norwegian Animal Research Authority. Twenty Duroc/ Norwegian Landbreed crossbred pigs (Sus scrofa) were bought from a commercial pig farm, from which mycobacteriosis had not been reported. Random sampling for bacteriological analysis of peat and sawdust used at the farm was completed, and mycobacteria were not detected. To further exclude the presence of $M$. avium in the herd, bacteriological examination of Lymphonodii (Lnn). mandibulares, Lnn. jejunales, Lnn. ileocolici, Lnn. colici, tonsils, spleens and Peyer's patches of jejunum and ileum collected from animals from the same farm at conventional slaughter was performed. No mycobacteria were detected. Organs from these un-inoculated animals were also used for macroscopic and microscopic comparison in the pathological examination of organs from the experimentally infected animals. Due to the limited housing facilities a formal negative control group held under identical conditions as the inoculated animals was not possible to include in the study. As the study was aiming at comparing two subspecies, such a control group was not considered necessary as long as the original herd was to the authors' best knowledge uninfected.

\section{Experimental design}

At the age of 6 weeks, the pigs to be inoculated were housed in two groups of ten at a research facility at the Norwegian Veterinary Institute. Between arrival at the research facility and inoculation, the animals were allowed one week of acclimatization. The two groups were evenly distributed with regards to weight, sex and litter origin, and kept in separate rooms, each accessible only through sluice chambers. Water was provided from a common drinking water system, from which no mycobacteria were detected by culture. The animals were fed commercially available pig feed and kept on a bedding of wood shavings, which is considered less likely to harbour mycobacteria, as opposed to sawdust [11].

The pigs were inoculated per os with $5 \times 10^{9}$ viable bacteria at the age of 7 weeks. Animals \#1 - \#10 received an isolate of Mah, and animals \#11 - \#20 an isolate of Maa. The inocula were mixed with raspberry jam intended for human consumption and applied at the back of the tongue. The animals had been trained for a week in advance to efficiently swallow treats applied in this manner.

Five pigs from each group of ten were euthanized under general anaesthesia for pathological and bacteriological examination at 6 weeks after inoculation, the remaining pigs at 12 weeks. Prior to euthanasia, infection was confirmed by IFN- $\gamma$ assays and tuberculin testing. One animal in the last group to be sacrificed was euthanized after 8 weeks, as its weight gain was unsatisfactory. General anaesthesia of the pigs was achieved by deep i.m. injection of xylazine $2 \mathrm{mg} / \mathrm{kg}$ (Rompun ${ }^{\mathbb{R}}$ vet. $20 \mathrm{mg} / \mathrm{ml}$, Bayer, Oslo, Norway), butorphanol $0.2 \mathrm{mg} / \mathrm{kg}$ (Dolorex ${ }^{\circledR}$ vet. 10 $\mathrm{mg} / \mathrm{ml}$, Intervet/Shering Plough Animal Health, Bergen, Norway) and ketamine $15 \mathrm{mg} / \mathrm{kg}$ (Ketalar ${ }^{\circledR} 50 \mathrm{mg} / \mathrm{ml}$, Pfizer, Oslo, Norway) prior to euthanization by the i.v. administration of $200 \mathrm{mg} / \mathrm{kg}$ pentobarbital (Pentobarbital $10 \%$, NAF Apotek, Oslo). Gross pathological examination and sampling was performed immediately post mortem.

\section{Preparation of bacterial inoculum}

Two Norwegian clinical isolates of M. avium, VI101 and 1794, were used in the study. VI101, a smooth and opaque $M a h$ isolate, originates from a porcine cervical lymph node with pathological lesions detected at slaughter. 1794 is a rough $\mathrm{Maa}$ isolate from a Norwegian Rough-legged Buzzard (Buteo lagopus) diagnosed with avian tuberculosis. Data on isolation, restriction fragment length polymorphism (RFLP) type and biofilm producing abilities for both isolates were presented by Johansen et al. 2007 and 2009 [17,30].

Bacterial inocula were prepared from seven days subcultures grown on plates of Middlebrook 7H10 (BD 
Diagnostics, Sparks MD) with 10\% OADC (BD Diagnostics) at $37^{\circ} \mathrm{C}$. For adjusting the number of bacteria in the inoculum, real time PCR on the single copy housekeeping gene $h s p 65$ [31] was performed on serial dilutions of a suspension adjusted to McFarland standard 2.0, homogenised through a 23G needle. Diluents were HBSS (Invitrogen, Oslo, Norway) in cellular assays and MQ water in animal assays. To confirm the real time PCR results, the dilutions were additionally seeded onto Middlebrook 7H10 (BD Diagnostics) and incubated at $37^{\circ} \mathrm{C}$ for 2 weeks for CFU count.

\section{Macrophage infection assay}

Peripheral blood mononuclear cells (PBMCs) were separated by density gradient centrifugation from $50 \mathrm{ml}$ of heparinised blood from four non-inoculated pigs at the original farm. Summarized, $2 \times 25 \mathrm{ml}$ blood was layered on top of $2 \times 10 \mathrm{ml}$ Lymfoprep (Axis-Shield, Oslo, Norway) and centrifuged at $400 \mathrm{~g}$ for $30 \mathrm{~min}$, followed by washing three times. The cells were resuspended in $50 \mathrm{ml}$ RPMI-1640 with sodium bicarbonate (Sigma-Aldrich, Oslo, Norway) and 1\% L-glutamine (Sigma-Aldrich) at a concentration of $3.5 \times 10^{6}$ PBMCs per $\mathrm{ml}$. One $\mathrm{ml}$ of cell suspension was added to each well of 12 well cell culture plates (Sigma-Aldrich), giving $3.5 \times 10^{5}$ monocytes per well. To isolate adherent cells, the plates were incubated at $37^{\circ} \mathrm{C}$ in $0.5 \% \mathrm{CO}_{2}$ for one hour, followed by two steps of washing with pre-warmed medium to remove lymphocytes. The cells were incubated further for one week in medium with $10 \%$ swine serum (SS) (Rockland Immunochemicals Inc., Gilbertsville, PA) to mature the monocytes into macrophages. Every second day $0.5 \mathrm{ml}$ of the supernatant was carefully replaced with fresh medium. The seven days old macrophage cultures were infected with a multiplicity of infection (MOI) of 10:1 bacteria per macrophage, as determined by $h s p 65$ real time PCR. Macrophages from all four pigs were infected in duplicate wells with Mah and Maa, respectively. After six hours of incubation, the wells were washed vigorously three times with medium to remove any extracellular bacteria, and incubated further. Cell lysis was performed in $1.5 \mathrm{ml} \mathrm{MQ}$ water. Lysates were harvested from both infected and uninfected control wells after six hours, and after one and seven days of infection and stored at $-20 \mathrm{C}$ in $2 \mathrm{ml}$ O-ring vials (Biospec Products Inc., Techtum Lab. Umeå, Sweden) containing $200 \mu \mathrm{l} 0.1 \mathrm{~mm}$ silica beads (Biospec Products Inc.). After completion of sampling, lysates were thawed, inactivated at $100^{\circ} \mathrm{C}$ for $20 \mathrm{~min}$, bead beaten and analysed by $h s p 65$ real time PCR for determination of the extent of intracellular replication [31,32].

\section{Immunological testing}

All animals were tested for IFN- $\gamma$ response to purified protein derivate from $M$. avium (PPDa) at 5 weeks, and eight of the remaining pigs at 11 weeks. Briefly, one $\mathrm{ml}$ of heparinised blood was stimulated overnight in 5\% $\mathrm{CO}_{2}$ at $37^{\circ} \mathrm{C}$ with PPDa or PBS as negative control. Plasma was harvested and stored at $-70 \mathrm{C}$ until analysed, using a commercial IFN- $\gamma$ swine ELISA kit (Invitrogen) according to the manufacturer's recommendations.

Tuberculin skin test was performed before euthanasia, after blood sampling for IFN- $\gamma$ testing was completed. Inhouse produced PPDa from Mah D4 and PPDt from pooled M. tuberculosis strains E9655 and E5 were injected intradermally behind respectively left and right ear of the animals at a dose of $0.1 \mathrm{ml}$. The thickness of the skin was measured before and $72 \mathrm{~h}$ after injection. Increased measures and occurrence of visible changes in the skin were recorded. Any visible oedema or redness or a $>2 \mathrm{~mm}$ increase of the skin thickness at the injection site was regarded as a positive reaction of delayed hypersensitivity to PPD.

\section{Gross pathology and histopathology}

The animals were successively euthanized and placed on a table covered in plastic. A thorough post mortem examination was performed immediately after euthanasia. The table was disinfected and the cover changed between the animals. Incisions were made on a selection of lymph nodes and organs, in order to reveal potential macroscopic lesions compatible with mycobacterial infection, which were defined as distinct foci of white-yellow tissue, further addressed as tuberculous lesions. A standardized sampling procedure was performed on tonsils, the medial lung lobe, the dorsal end of the spleen, the right medial liver lobe, jejunal and ileal Peyer's patches, Lnn. mandibulares, Lnn. bifurcationis sinn., and the gastrointestinal lymph nodes Lnn. jejunales, Lnn. ileocolici and Lnn. colici in each animal. Each tissue sample was subjected to histopathological and bacteriological examination. Five grams of faeces were collected at necropsy from each inoculated pig. Gloves, scalpels and cutting boards were changed between each sampling, to avoid cross contamination between organs and animals.

Tissues for light microscopy were fixed in phosphatebuffered $4 \%$ formaldehyde, prior to routine processing and paraffin embedding, followed by sectioning at 3-4 $\mu \mathrm{m}$ and staining with hematoxylin-eosin (HE) and Ziehl-Neelsen $(\mathrm{ZN})$ to facilitate the detection of acid-fast bacteria. An additional section of those samples indicative of fibrous tissue were stained with van Gieson (vG) and samples indicative of mineralisation were stained with von Kossa. On microscopy, granulomatous lesions were classified into three categories according to the system described by Hibiya et al. [33]. However, the classification system was slightly simplified, due to the wide range of variations within the histopathological appearance. In the present study, the term exudative was applied on unencapsulated 
lesions ranging from the mere presence of single multinucleated giant cells and/or a few macrophages to the larger, well-circumscribed granulomas consisting of epitheloid cells and various numbers of lymphoid cells, multinucleated giant cells, neutrophils and eosinophils. Reactions were classified as proliferative when the lesions were partially or fully surrounded by fibroblasts and showing various degrees of encapsulation, from loosely woven connective tissue to thick collagen capsules. Sections with the simultaneous occurrence of exudative and proliferative reactions were classified as mixed granulomas. Necrosis and calcification could occur within all the categories.

All sections presenting multinucleated giant cells and granulomatous lesions were examined for porcine circovirus 2 (PCV2) by immunohistochemical staining, since PCV2 is a common differential diagnose to mycobacteriosis when granulomatous reactions are observed in pigs [34]. Briefly, after clearing with xylene, sections were rehydrated and stained with a monoclonal mouse anti-PCV2 antibody (mAb F1217-2C6 H9 A2) at dilution 1:20 000. The antibody was kindly provided by Dr. Gordon Allan, Department of Agriculture for Northern Ireland, Veterinary Science Division, Belfast, UK. PCV2 was not detected in any samples.

\section{Bacteriological examination}

Defined amounts of tissue and faecal material were decontaminated as previously described [35]. Briefly, 1-3 g of the sample were homogenised in saline, decontaminated with $5 \%$ oxalic acid with $0.1 \%$ malachite green and centrifuged, followed by resuspension of the pellet in saline and inoculation on slants of Middlebrook 7H10 medium (BD Diagnostics) with $10 \%$ OADC (BD Diagnostics) with and without antibiotics (final concentrations of $100 \mu \mathrm{g} / \mathrm{ml} \mathrm{car-}$ benicillin, $200 \mathrm{U} / \mathrm{ml}$ polymyxin B sulphate, $19.5 \mu \mathrm{g} / \mathrm{ml}$ trimethoprim lactate and $10 \mu \mathrm{g} / \mathrm{ml}$ amphotericin B), Petragnani's medium (BD Diagnostics), Stonebrink's medium (BD Diagnostics) and Loewenstein-Jensen's egg-based medium (BD Diagnostics). An additional step of decontamination with $\mathrm{NaOH}$ prior to the step of treatment with oxalic acid with malachite green was performed on faecal samples. The media were incubated for 8 weeks at $37^{\circ} \mathrm{C}$ and observed for growth on a weekly basis. Cultures of acid-fast bacteria were further identified by IS1245 RFLP [17], IS901 PCR (primer 901a and 901c) [36] and IS1245 PCR (primer p40 and p41) [37] to confirm the expected subspecies and RFLP profile and thereby rule out cross contamination.

For real time PCR, template DNA was extracted from tissue samples by using NucliSENS ${ }^{\circledR}$ easyMag ${ }^{\circledR}$ (Biomérieux, Marcy l'Etoile, France). Briefly, $0.1 \mathrm{ml}$ of the saline used for overnight soaking of the sample homogenate was transferred to $1 \mathrm{ml} \mathrm{NucliSENS^{ \circledR }}$ easyMag $^{\circledR}$ lysis buffer (Biomérieux) and inactivated at $80^{\circ} \mathrm{C}$ for $20 \mathrm{~min}$ prior to
2 min bead beating with $0.2 \mathrm{ml}$ of $0.1 \mathrm{~mm}$ silica beads (Biospec Products Inc.), followed by DNA extraction by the generic protocol. Real time PCR was performed on the template DNA by amplification of a $82 \mathrm{bp}$ target sequence of the insertion element IS1245, as described by Agdestein et al. [20]. Samples were regarded as positive when $\mathrm{Ct} \leq 38$.

\section{Statistical analysis}

Differences between the groups were assessed by using a Wilcoxon-Mann Whitney non-parametric test. $P \leq 0.05$ was considered statistically significant.

\section{Results}

\section{Clinical signs}

Animal \#15 and \#18, both inoculated with Maa, had diarrhea for five and two days, respectively, starting three days after infection. Animal \#18 developed a cough, which was later explained by eosinophilic interstitial pneumonia detected at pathological examination, considered unrelated to mycobacterial infection. Animal \#6, inoculated with Mah, showed a decrease in growth rate and was therefore euthanized and subjected to necropsy at week eight, upon which no macroscopic lesions compatible with mycobacteriosis were detected. The remaining animals did not show any clinical signs of infection.

\section{Immunological testing}

To confirm the infection, the animals were tested in an IFN- $\gamma$ assay and the tuberculin test. All animals were negative in the IFN- $\gamma$ assay before inoculation, while after 5 weeks, samples from eight (40\%) out of the infected animals produced IFN- $\gamma$ in response to PPDa. Of these, five were infected with $M a h$, and three with Maa. All inoculated animals tested at 11 weeks after infection, showed a strong IFN- $\gamma$ response to PPDa, and there was no significant difference between animals in the Mah and Maa groups. Positive reactions on tuberculin skin testing were seen in all infected groups, but not in every individual. The strongest reactions were seen at 11 weeks, in both groups. Ulcerations or necrosis of the skin were observed in six animals. Three of the animals tested at 5 weeks showed no reaction to either types of PPD used. One of these pigs was infected with Mah, the other two with Maa.

\section{Macrophage infection assay}

Both Mah and Maa were able to infect and replicate within macrophages, however some differences between the subspecies were observed. Maa showed a greater ability of invasion and replication within porcine macrophages at all sampled points in time (Figure 1). The most prominent difference was observed at day seven. 


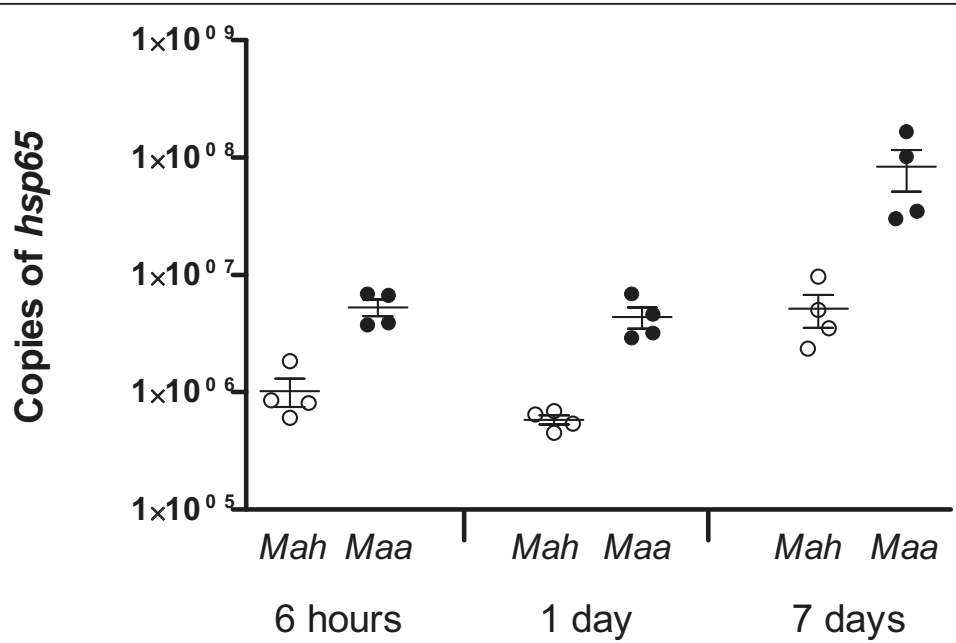

Figure 1 Replication of Mycobacterium avium subsp. hominissuis (Mah) or Mycobacterium avium subsp. avium (Maa) in porcine macrophages. Intracellular replication in macrophages from four un-inoculated pigs was measured by the number of the single copy housekeeping gene hsp65 copies, as determined by real time PCR analysis of lysates harvested at six hours, one day and seven days after infection. Numbers are presented as the mean of duplicate wells. Control lysates were negative on real time PCR and are not shown.

\section{Gross pathology and histopathology}

Macroscopic lesions compatible with mycobacterial infection were found in six animals (Table 1), ranging from one to four $\mathrm{mm}$ in size. The majority of macroscopic lesions were seen in mesenteric lymph nodes. In the animals sampled at week six, visible tuberculous lesions were detected only in one animal, which was inoculated with Maa. At 12 weeks, 10 samples from three animals in the Mah group and three samples from two animals in the Maa group, showed tuberculous lesions on macroscopic examination. Only two $L n n$. mandibulares, both from pigs inoculated with Mah, had visible tuberculous lesions. There were no visible tuberculous lesions in tonsils, spleens, Lnn. bifurcationis sinn., nor in the lungs in any of the animals. Crater-like changes of four mm were seen in the jejunal Peyer's patches of animals \#18 and \#19, inoculated with Maa.

Additionally, on gross examination cystic changes of two to $15 \mathrm{~mm}$ were detected in 10 lymph node samples originating from six different animals in the Maa group. Only two of these samples had additional macroscopic lesions in the surrounding tissue. However, all samples with cystic changes, except one, were accompanied by either the presence of acid-fast rods on histopathological examination or positive results from culture or real time PCR The fluid content within the cystic compartments ranged from transparent to opaque and proved sterile on general bacteriologic examination. To the authors best knowledge the number of cystic lesions of lymph nodes reported previously in pigs is negligible, and cysts have not been described in association with mycobacterial disease in swine. To determine whether or not mycobacteria were directly responsible for the cystic changes, further investigations are required.

On histopathological examination granulomatous lesions were seen in 32 out of 110 (29\%) standardized tissue samples from pigs inoculated with $M a h$, and acidfast bacilli were detected in $20(60 \%)$ of these. In pigs inoculated with $M a a$, granulomatous lesions were detected in 30 out of 110 (27\%) samples, nine containing acid-fast bacilli (Tables 1 and 2). The majority of the lesions were classified as exudative, while encapsulated granulomas were always seen together with exudative lesions, and the samples therefore classified as mixed. Six samples from the Mah group and two samples from the Maa group were of the mixed type, and they were all found in gastrointestinal lymph nodes. One animal infected with Maa had ulceration of the mucosa in addition to exudative reaction in both samples from the Peyer's patches.

Histopathological lesions were seen in every type of organ examined, but most frequently in gastrointestinal lymph nodes, Lnn. mandibulares, ileal Peyer's patches, tonsils, and jejunal Peyer's patches (Table 2). Lesions were found in $43 \%$ of the gastrointestinal lymph node samples in the Mah group and $23 \%$ in the Maa group. Calcification and necrosis were seen in gastrointestinal lymph nodes 12 weeks after infection with both subspecies, seven in animals infected with Mah and three in the Maa group. With regards to Lnn. mandibulares and tonsils, the number of samples with histopathological lesions, differed marginally between the two groups. In total, nine samples of Lnn. mandibulares and seven tonsils had histopathological lesions. Necrosis was observed 


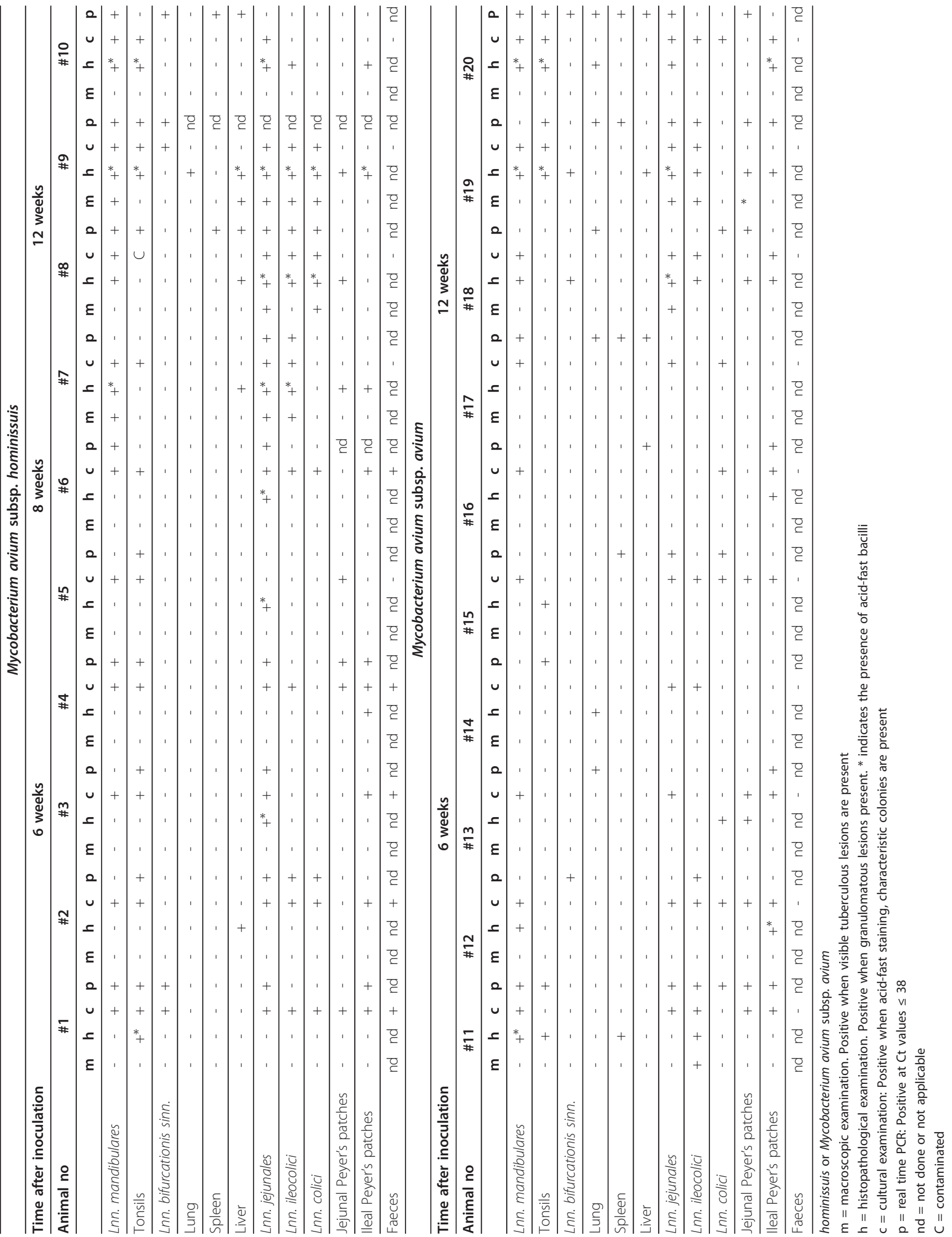




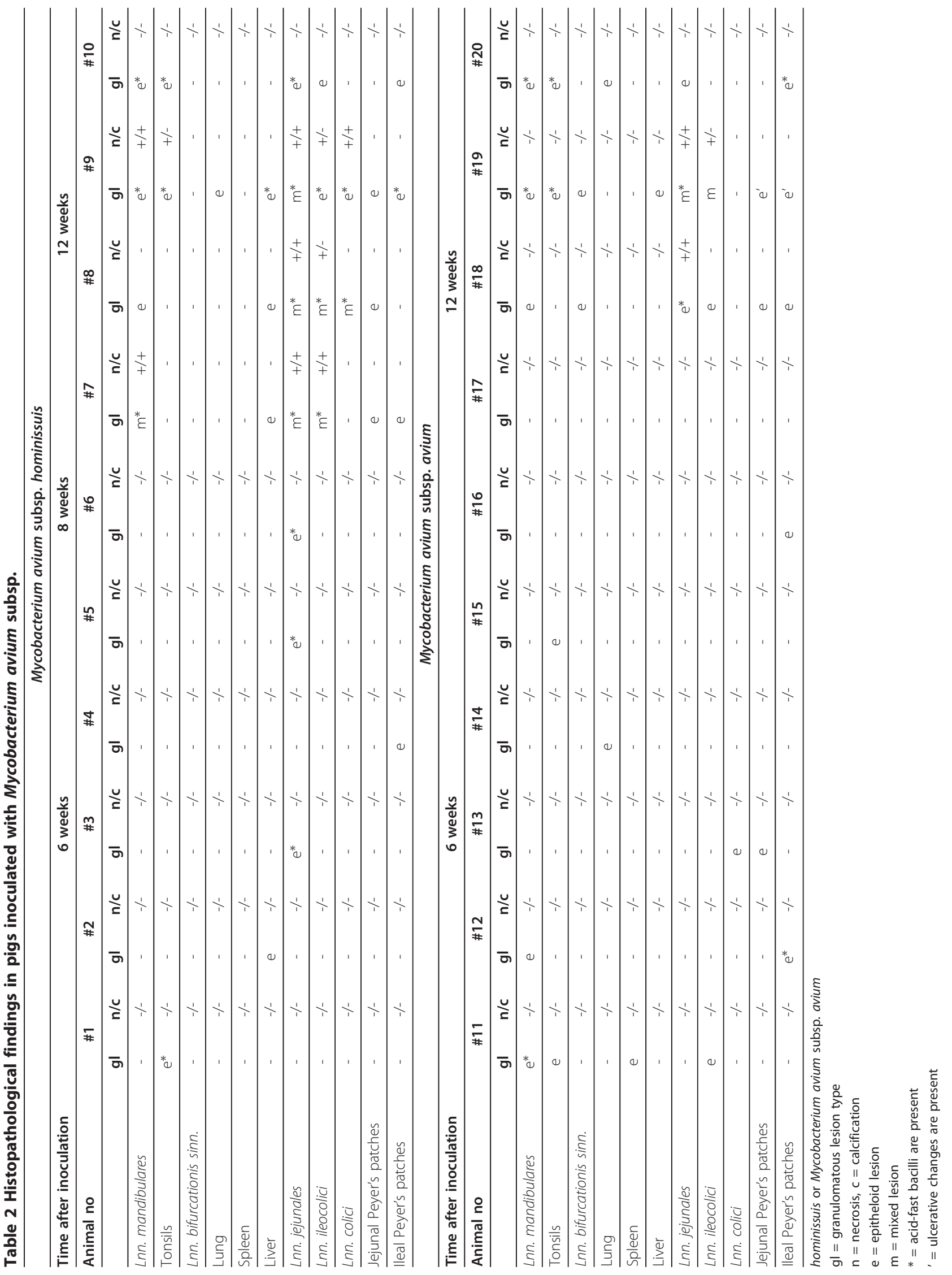


in one tonsil and two Lnn. mandibulares from the Mah group, of which one was calcified.

On three occasions multinucleated giant cells were observed in samples from un-inoculated animals, indicating early granuloma formation. However, acid-fast bacilli and PCV2 virus were not detected, and infection is not strictly required for these changes to arise [38]. There might be unspecific reasons for the presence of multinucleated giant cells.

\section{Bacteriological examination}

After 6 weeks of infection, Mah was detected by culture from faecal samples from four out of five animals, while Maa was not detected in faecal samples from any animal (Tables 1 and 3). This difference between the groups proved to be significant $(P \leq 0.05)$. Mah was also cultured from faeces originating from animal \#6, which was sacrificed at week eight. Twelve weeks after infection, none of the faecal samples were positive on culture.

Mycobacteria were retrieved by culture from each of the infected animals, showing different tissues involved (Tables 1 and 3). In total, 50 organ samples from pigs infected with Mah and 43 samples from the Maa infected pigs contained live mycobacteria. IS1245 RFLP, IS901 PCR and IS1245 PCR confirmed that the isolated mycobacteria were of the same subspecies as the isolates used for inoculation, and that the RFLP profiles were unchanged. The distribution of positive samples was similar between the groups of subspecies and duration

Table 3 Mycobacterial growth in tissues and faeces from Mycobacterium avium subsp. hominissuis or Mycobacterium avium subsp. avium inoculated pigs

\begin{tabular}{|c|c|c|c|c|c|c|c|c|c|c|}
\hline \multicolumn{11}{|c|}{ Mycobacterium avium subsp. hominissuis } \\
\hline \multirow{2}{*}{$\begin{array}{l}\text { Time after inoculation } \\
\text { Animal no }\end{array}$} & \multicolumn{4}{|c|}{6 weeks } & \multicolumn{2}{|r|}{8 weeks } & \multicolumn{4}{|c|}{12 weeks } \\
\hline & $\# 1$ & \#2 & $\# 3$ & \#4 & $\# 5$ & \#6 & $\# 7$ & \#8 & \#9 & $\# 10$ \\
\hline Lnn. mandibulares & 4 & 1 & 4 & 3 & 4 & 3 & 1 & 3 & 4 & 4 \\
\hline Tonsils & 4 & 1 & 4 & 4 & 4 & 4 & 1 & C & 4 & 4 \\
\hline Lnn. bifurcationis sinn. & 1 & - & - & - & - & - & - & - & 2 & - \\
\hline Lung & - & - & - & - & - & - & - & - & - & - \\
\hline Spleen & - & - & - & - & - & - & - & - & - & - \\
\hline Liver & - & - & - & - & - & - & - & - & - & - \\
\hline Lnn. jejunales & 4 & 4 & 4 & 4 & - & 2 & 4 & 4 & 4 & 4 \\
\hline Lnn. ileocolici & 3 & 3 & - & 2 & - & 1 & 2 & 4 & 4 & - \\
\hline Lnn. colici & 4 & 4 & - & - & - & 4 & - & 4 & 4 & - \\
\hline Jejunal Peyer's patches & 1 & - & - & 4 & 2 & - & - & - & - & - \\
\hline Ileal Peyer's patches & 4 & 1 & 2 & 2 & - & 3 & - & - & - & - \\
\hline Faeces & 4 & 1 & 3 & 2 & - & 2 & - & - & - & - \\
\hline \multicolumn{11}{|c|}{ Mycobacterium avium subsp. avium } \\
\hline Time after inoculation & \multicolumn{5}{|c|}{6 weeks } & \multicolumn{5}{|c|}{12 weeks } \\
\hline Animal no & \#11 & \#12 & $\# 13$ & $\# 14$ & $\# 15$ & \#16 & $\# 17$ & \#18 & \#19 & \#20 \\
\hline Lnn. mandibulares & 3 & 2 & 1 & - & 1 & 1 & 1 & 4 & 4 & 4 \\
\hline Tonsils & - & - & - & - & - & - & - & - & 1 & 1 \\
\hline Lnn. bifurcationis sinn. & - & - & - & - & - & - & - & - & - & - \\
\hline Lung & - & - & - & - & - & - & - & - & - & - \\
\hline Spleen & - & - & - & - & - & - & - & - & - & - \\
\hline Liver & - & - & - & - & - & - & - & - & - & - \\
\hline Lnn. jejunales & 4 & 2 & 1 & 2 & 1 & - & 1 & 4 & 4 & 2 \\
\hline Lnn. ileocolici & 1 & 1 & - & 2 & 1 & - & - & 3 & 4 & 4 \\
\hline Lnn. colici & - & 1 & - & - & 1 & 1 & 2 & - & - & 2 \\
\hline Jejunal Peyer's patches & 4 & 2 & 1 & - & 3 & - & - & - & - & - \\
\hline Ileal Peyer's patches & 3 & 2 & 3 & - & 3 & 1 & - & 1 & - & 1 \\
\hline Faeces & - & - & - & - & - & - & - & - & - & - \\
\hline
\end{tabular}


of infection. At 6 weeks of infection, the number of culture positive samples per animal of the Mah group ranged from three to eight out of 11, and in the Maa group from two to six. At 12 weeks, the range of positive samples per animal was three to six in both groups. Although not significantly different, there is a tendency of higher CFU counts in the samples from pigs inoculated with Mah, compared to Maa. More than $100 \mathrm{CFU}$ were detected in 29 tissue samples from Mah infected pigs and in nine from the Maa group. Only eight samples from Mah infected pigs contained one to nine CFU, compared to 19 in the Maa group (Table 3).

All Lnn. mandibulares, except from one animal infected with Maa, were positive on culture. All tonsils from the animals in the Mah group were positive, except from one sample that was unreadable due to contamination by other bacteria. Only two tonsil samples were positive in the Maa group, both collected at 12 weeks (Tables 1 and 3 ). In total, the difference between bacterial growth from tonsil samples was significant between the two subspecies.

Live mycobacteria were detected in the gastrointestinal lymph nodes in all but one animal, which belonged to the Mah group sacrificed at 6 weeks. Bacteria were most frequently found in the jejunal lymph nodes. In addition, seven out of ten samples from jejunal or ileal Peyer's patches were positive on culture 6 weeks after infection in the Mah group. However, in the Mah group sacrificed at 12 weeks, all samples from the Peyer's patches were negative. In the Maa group sacrificed at 6 weeks, eight out of ten samples from Peyer's patches were positive, dropping to three out of ten after 12 weeks.

Mycobacteria were not found by culture from lungs, liver and spleen. However, live mycobacteria were detected in Lnn. bifurcationis sinn. in two animals of the Mah group, one sacrificed at 6 weeks and the other at 12 weeks.

DNA containing IS1245 was detected in tissue samples from all inoculated individuals by real time PCR (Table 1).

\section{Discussion}

Infection with the $M$. avium subspecies was established in all inoculated animals, as shown by positive results in several of the analyses performed. This indicated that both Mah and Maa had the potential to establish infection in pigs, and once infected with either subspecies, the course of infection seemed to be quite similar. There was a significant difference between the animals infected with Maa and those infected with Mah with regards to faecal shedding. Whereas live Mah was profoundly excreted in faeces 6 weeks after inoculation, Maa was not detected in faecal samples. Although earlier events of shedding in the Maa infected pigs cannot be excluded, this observation points at a greater ability of Mah to cause an increased load of mycobacteria in the environment and an intensified infection pressure in the pig herd. Faecal shedding of $M$. avium in pigs has been described in the older literature by various authors [23-25]. Jørgensen et al. [23] demonstrated this event in the Maa strain ATCC 25291, with subsequent infection of contact animals. However, the following lack of excretion of mycobacteria from the contact animals led the authors to generally reject a faecal-oral route of transmission for $M$. avium. It is important to consider the fact that Jørgensen et al. used a laboratory strain whose properties might have been modified by numerous passages, and that the study involved $M a a$ only. In the present study, the animals were inoculated with clinical isolates of Maa and Mah at comparable doses and kept under the same conditions, enabling the comparison between the subspecies.

In 1964, Kauker and Zettl [26] performed a comparative study with experimental infection in pigs using one mycobacterial isolate from a porcine lymph node and another from a bird, presumably Mah and Maa, respectively. Infection of contact animals was only seen in the group infected with the pig isolate, something that supports the hypothesis of differences between Mah and Maa with regards to transmission through faecal shedding. However, more recent papers postulate that Mah is not transmitted between pigs, due to the diversity of IS1245 RFLP profiles of isolates originating from the same farm [10,17]. Isolates of identical RFLP profiles have indeed been detected in pigs bred at the same facility $[14,15,20]$. The current findings propose that the route of faecal-oral infection in pigs is more pronounced in Mah than in Maa, which might be one reason for the higher incidence of the prior subspecies in the porcine population. One might hypothesize that Maa infection in pigs mainly occurs as individual events through ingestion of material containing mycobacteria, such as bird shedding or peat [7], whereas Mah additionally has a greater ability to keep circulating in the pig herd once introduced. Additionally, Mah is proposed to be the only true environmental subspecies of M. avium [2], suggesting a better ability of Mah than Maa to replicate in the environment. There is no doubt that Mah can be introduced to pig herds by contaminated peat and sawdust, still transmission from pig to pig also seems to occur.

The most obvious finding of the present study with regards to differences in the count of live bacteria in organs was done in tonsils, where Mah proved to be present in considerable amounts, while Maa was only sporadically detected. The tonsils are a proposed reservoir for the faecal-oral route of transmission [25] and 
strengthens the hypothesis of Mah being more likely than Maa to spread by pig-to-pig contact.

The amount of mycobacteria and the numbers and severity of tuberculous lesions detected in the present study was somewhat greater in pigs inoculated with Mah than with Maa, although not significantly. This seems to be in contrast to the study of Kauker and Zettl [26], who found the bird isolate to be hypervirulent, causing systemic disease and death, while the pig isolate only gave rise to lymph node lesions. One should bear in mind that Kauker and Zettl had only two infected animals and one control- and contact animal per group, which makes it difficult to draw any conclusions.

The extent of intracellular replication in cell cultures is widely used as a measure for mycobacterial virulence [39-41]. Results from the present infection assays in porcine macrophage cultures suggested that Maa replicated intracellularly to a greater extent than Mah, thus defining it as more virulent by the prevailing standards. The results from the cell assay support the assumption that the low prevalence of mycobacteriosis caused by Maa in the pig population is not due to lack of virulence or failure to establish infection, which was confirmed by the experimental infection in pigs. However, the contradictory results of the present in vitro and in vivo studies, suggests that assaying virulence of facultative pathogen mycobacteria based only on in vitro studies should be done with some caution.

In the present study, mycobacteria were mainly detected in lymph nodes, tonsils and intestinal segments, which have previously been reported as typical locations [4]. In concordance with other studies $[10,18]$, massive occurrence of live mycobacteria and exudative histopathological lesions not accompanied by gross lesions were findings in a majority of samples. In Lnn. mandibulares, macroscopic lesions characteristic of mycobacterial infection were only detected in two of the pigs, even though mycobacteria could be detected by culture from various organs in all pigs. This should be of interest with regards to food safety, as the gross examination of Lnn. mandibulares plays an important role in the detection of mycobacterial infection in slaughtered pigs.

The present study indicated that Maa has an infection potential in pigs on almost the same level as Mah, leaving differences in exposure or sensitivity of detection at slaughter as probable reasons for the fact that Maa has not been reported in the Norwegian swine population. In some countries, Maa has been detected in pigs [7,8], something that might be explained by differences in housing. Pig housing that allows birds to enter, or the keeping of free range pigs, presumably enables an increased infection pressure of Maa. In Norway, pig farms are mainly closed in-door facilities, and Maa infections in the Norwegian bird population are rarely diagnosed (B. Djønne, NVI, personal communication). Maa has also sporadically been detected in peat [18], which leaves regional differences in peat processing as an additional explanation for the fact that some countries are more troubled with Maa infection in pigs than others [7].

\section{Conclusions}

The present study re-introduces a hypothesis of a faecaloral route of infection being a more prominent feature in Mah than in Maa. This might contribute to the fact that Mah is the more commonly detected subspecies in pigs. Additionally, low exposure to infected birds might explain the absence of Maa infection in pigs in many countries. The observations of the present study also propose that the higher reported incidence of infection with Mah than Maa in the pig population might be due to the formation of more obvious lesions in the prior. Nevertheless, future assays of experimental infection with contact animals of young age and other clinical isolates of Maa and Mah are needed to mimic the situation in swine farms and to conclude on behalf of the subspecies with regards to different strategies of transmission and virulence.

\section{Acknowledgements}

The project was funded by the Research Council of Norway and the Norwegian Veterinary Institute. Birthe Graeber at the NVI animal facility and Ingunn Ruud at Section of Pathology are greatly acknowledged for eminent technical assistance, together with the staff at Section of Immunoprophylaxis, Section of bacteriology and Section of Pathology. Ari Ek at The Norwegian Food Safety Authority, District Office Ytre Østfold, deserves credit for enabling the collection of samples at slaughter. The farmer at the original facility is also appreciated for providing animals and technical assistance.

\section{Author details}

'Norwegian Veterinary Institute, PO. Box 750 Sentrum, N-0106 Oslo, Norway. ${ }^{2}$ Norwegian Pig Health Service, Animalia, PO. Box 396 Økern, N-0513 Oslo, Norway.

\section{Authors' contributions}

AA contributed to conception and design of the experiment, sampling and bacteriological analysis, data analysis and drafting of the manuscript. TBJ contributed to conception and design of the experiment, sampling and bacteriological analysis and drafting of the manuscript. ØK contributed to necropsy and histopathological examination and writing of the manuscript. AJ contributed with advice on pig husbandry and to collection of blood samples and writing of the manuscript. BD contributed to conception and design, bacteriological analysis and drafting of the manuscript. 10 contributed to conception and design of the experiment, sampling, immunological analysis and drafting of the manuscript. All authors read and approved the final manuscript.

\section{Competing interests}

The authors state that there are no competing interests related to the present study.

Received: 12 September 2011 Accepted: 27 January 2012

Published: 27 January 2012 


\section{References}

1. Inderlied CB, Kemper CA, Bermudez LE: The Mycobacterium avium complex. Clin Microbiol Rev 1993, 6:266-310.

2. Turenne $C Y$, Wallace R Jr, Behr MA: Mycobacterium avium in the postgenomic era. Clin Microbiol Rev 2007, 20:205-229.

3. Biet F, Boschiroli ML, Thorel MF, Guilloteau LA: Zoonotic aspects of Mycobacterium bovis and Mycobacterium avium-intracellulare complex (MAC). Vet Res 2005, 36:411-436.

4. Thorel MF, Huchzermeyer HF, Michel AL: Mycobacterium avium and Mycobacterium intracellulare infection in mammals. Rev Sci Tech 2001, 20:204-218.

5. Thorel MF, Huchzermeyer H, Weiss R, Fontaine JJ: Mycobacterium avium infections in animals. Literature Review Vet Res 1997, 28:439-447.

6. Falkinham JO III: Epidemiology of infection by nontuberculous mycobacteria. Clin Microbiol Rev 1996, 9:177-215.

7. Pavlik I, Svastova P, Bartl J, Dvorska L, Rychlik I: Relationship between IS901 in the Mycobacterium avium complex strains isolated from birds, animals, humans, and the environment and virulence for poultry. Clin Diagn Lab Immunol 2000, 7:212-217.

8. Mobius P, Lentzsch P, Moser I, Naumann L, Martin G, Kohler H: Comparative macrorestriction and RFLP analysis of Mycobacterium avium subsp. avium and Mycobacterium avium subsp. hominissuis isolates from man, pig, and cattle. Vet Microbiol 2006, 117:284-291.

9. Gwatkin R, Mitchell CA: Avian tuberculosis infection in Swine. Can J Comp Med Vet Sci 1952, 16:345-347.

10. Komijn RE, de Haas PE, Schneider MM, Eger T, Nieuwenhuijs JH, van den Hoek RJ, Bakker D, van Zijd Erveld FG, Van Soolingen D: Prevalence of Mycobacterium avium in slaughter pigs in The Netherlands and comparison of IS1245 restriction fragment length polymorphism patterns of porcine and human isolates. J Clin Microbiol 1999, 37:1254-1259.

11. Matlova L, Dvorska L, Palecek K, Maurenc L, Bartos M, Pavlik I: Impact of sawdust and wood shavings in bedding on pig tuberculous lesions in lymph nodes, and IS1245 RFLP analysis of Mycobacterium avium subsp. hominissuis of serotypes 6 and 8 isolated from pigs and environment. Vet Microbiol 2004, 102:227-236.

12. Mijs W, de Haas P, Rossau R, Van der Laan T, Rigouts L, Portaels F, Van Soolingen D: Molecular evidence to support a proposal to reserve the designation Mycobacterium avium subsp. avium for bird-type isolates and ' $M$. avium subsp. hominissuis' for the human/porcine type of $M$. avium. Int J Syst Evol Microbiol 2002, 52:1505-1518.

13. Thegerstrom J, Marklund BI, Hoffner S, Axelsson-Olsson D, Kauppinen J, Olsen B: Mycobacterium avium with the bird type IS1245 RFLP profile is commonly found in wild and domestic animals, but rarely in humans. Scand J Infect Dis 2005, 37:15-20.

14. Domingos M, Amado A, Botelho A: IS1245 RFLP analysis of strains of Mycobacterium avium subspecies hominissuis isolated from pigs with tuberculosis lymphadenitis in Portugal. Vet Rec 2009, 164:116-120.

15. Wellenberg GJ, de Haas PE, van Ingen J, Van Soolingen D, Visser IJ: Multiple strains of Mycobacterium avium subspecies hominissuis infections associated with aborted fetuses and wasting in pigs. Vet Rec 2010, 167:451-454

16. Ritacco V, Kremer K, Van der Laan T, Pijnenburg JE, de Haas PE, van Soolingen D: Use of IS901 and IS1245 in RFLP typing of Mycobacterium avium complex: Relatedness among serovar reference strains, human and animal isolates. Int J Tuberc Lung Dis 1998, 2:242-251.

17. Johansen TB, Olsen I, Jensen MR, Dahle UR, Holstad G, Djonne B: New probes used for IS1245 and IS1311 restriction fragment length polymorphism of Mycobacterium avium subsp. avium and Mycobacterium avium subsp. hominissuis isolates of human and animal origin in Norway. BMC Microbiol 2007, 7:14

18. Matlova L, Dvorska L, Ayele WY, Bartos M, Amemori T, Pavlik I: Distribution of Mycobacterium avium complex isolates in tissue samples of pigs fed peat naturally contaminated with mycobacteria as a supplement. J Clin Microbiol 2005, 43:1261-1268

19. Alvarez J, Castellanos E, Romero B, Aranaz A, Bezos J, Rodriguez S, Mateos A, Dominguez $L$, de Juan $L$ : Epidemiological investigation of a Mycobacterium avium subsp. hominissuis outbreak in swine. Epidemiol Infect 2011, 139:143-148

20. Agdestein A, Johansen TB, Polacek V, Lium B, Holstad G, Vidanovic D, Aleksic-Kovacevic S, Jorgensen A, Zultauskas J, Nilsen SF, et al: Investigation of an outbreak of mycobacteriosis in pigs. BMC Vet Res 2011, 7:63.
21. Aronson T, Holtzman A, Glover N, Boian M, Froman S, Berlin OG, Hill H, Stelma G Jr: Comparison of large restriction fragments of Mycobacterium avium isolates recovered from AIDS and non-AIDS patients with those of isolates from potable water. J Clin Microbiol 1999, 37:1008-1012.

22. Rastogi N, Legrand E, Sola C: The mycobacteria: An introduction to nomenclature and pathogenesis. Rev Sci Tech 2001, 20:21-54.

23. Jorgensen JB: Experimental infection with Mycobacterium avium, serotype 2, in pigs. 4. Contact infection from orally inoculated pigs. Acta Vet Scand 1978, 19:58-72.

24. Thoen CO, Johnson DW, Himes EM, Menke SB, Muscoplat CC: Experimentally induced Mycobacterium avium serotype 8 infection in swine. Am J Vet Res 1976, 37:177-181.

25. Ellsworth S, Kirkbride CA, Johnson DD: Excretion of Mycobacterium avium from lesions in the intestine and tonsils of infected swine. Am J Vet Res 1980, 41:1526-1530.

26. Kauker $\mathrm{E}$, Zettl K: Beitrag zur käsigen Lymphknotenentzündung der Schweine. Ber Münch tierärztl Wschr 1964, 77:173-176.

27. Jorgensen JB: Experimental infection with Mycobacterium avium serotype 2, 2. Oral infection with large doses of M. avium. Acta Vet Scand 1977, 18:545-558.

28. Garrido JM, Vicente J, Carrasco-Garcia R, Galindo RC, Minguijon E, Ballesteros C, Aranaz A, Romero B, Sevilla I, Juste R, et al: Experimental infection of Eurasian wild boar with Mycobacterium avium subsp. avium. Vet Microbiol 2010, 144:240-245

29. Slana I, Kaevska M, Kralik P, Horvathova A, Pavlik I: Distribution of Mycobacterium avium subsp. avium and $M$. a. hominissuis in artificially infected pigs studied by culture and IS901 and IS1245 quantitative realtime PCR. Vet Microbiol 2010, 144:437-443.

30. Johansen TB, Agdestein A, Olsen I, Nilsen SF, Holstad G, Djonne B: Biofilm formation by Mycobacterium avium isolates originating from humans, swine and birds. BMC Microbiol 2009, 9:159.

31. Baba K, Pathak S, Sviland L, Langeland N, Hoosen AA, Asjo B, Dyrhol-Riise AM, Mustafa T: Real-time quantitative PCR in the diagnosis of tuberculosis in formalin-fixed paraffin-embedded pleural tissue in patients from a high HIV endemic area. Diagn Mol Pathol 2008, 17:112-117.

32. Pathak S, Wentzel-Larsen T, Asjo B: Effects of in vitro HIV-1 infection on mycobacterial growth in peripheral blood monocyte-derived macrophages. Infect Immun 2010, 78:4022-4032.

33. Hibiya K, Kasumi Y, Sugawara I, Fujita J: Histopathological classification of systemic Mycobacterium avium complex infections in slaughtered domestic pigs. Comp Immunol Microbiol Infect Dis 2008, 31:347-366.

34. Segales J, Allan GM, Domingo M: Porcine circovirus diseases. Anim Health Res Rev 2005, 6:119-142.

35. Valheim M, Djonne B, Heiene R, Caugant DA: Disseminated Mycobacterium celatum (type 3 ) infection in a domestic ferret (Mustela putorius furo). Vet Pathol 2001, 38:460-463.

36. Ahrens P, Giese SB, Klausen J, Inglis NF: Two markers, IS901-IS902 and p40, identified by PCR and by using monoclonal antibodies in Mycobacterium avium strains. J Clin Microbiol 1995, 33:1049-1053.

37. Johansen TB, Djonne B, Jensen MR, Olsen I: Distribution of IS1311 and IS1245 in Mycobacterium avium subspecies revisited. J Clin Microbiol 2005, 43:2500-2502.

38. Baba Y, Nakayama H, Yasoshima A, Uetsuka K, Kumagai S, Doi K: Thymic granulomatous lesions in pigs. Vet Pathol 2006, 43:1037-1040.

39. Tateishi Y, Hirayama Y, Ozeki Y, Nishiuchi Y, Yoshimura M, Kang J, Shibata A, Hirata K, Kitada S, Maekura R, et al: Virulence of Mycobacterium avium complex strains isolated from immunocompetent patients. Microb Pathog 2009, 46:6-12.

40. Li YJ, Danelishvili L, Wagner D, Petrofsky M, Bermudez LE: Identification of virulence determinants of Mycobacterium avium that impact on the ability to resist host killing mechanisms. J Med Microbiol 2010, 59:8-16.

41. Jha SS, Danelishvili L, Wagner D, Maser J, Li YJ, Moric I, Vogt S, Yamazaki Y, Lai B, Bermudez LE: Virulence-related Mycobacterium avium subsp. hominissuis MAV_2928 gene is associated with vacuole remodeling in macrophages. BMC Microbiol 2010, 10:100.

doi:10.1186/1746-6148-8-11

Cite this article as: Agdestein et al: A comparative study of

Mycobacterium avium subsp. avium and Mycobacterium avium subsp. hominissuis in experimentally infected pigs. BMC Veterinary Research 2012 8:11. 